Studies in Perception \& Action XV

L. van Dijk \& R. Withagen (Eds.)

\title{
Modelling and Simulating Action Dynamics in Underconstrained Tasks in Virtual Reality
}

\author{
Patric C. Nordbeck ${ }^{1}$, Maurice Lamb ${ }^{1}$, Paula Silva ${ }^{1}$ \\ ${ }^{1}$ Department of Psychology, Center for Cognition, Action, \& Perception, \\ University of Cincinnati.
}

Bernstein (1967) defined dexterity as the ability to consistently achieve a desired level of task performance across a wide variety of contextual conditions. This ability implicates adaptability of action patterns to both the requirements of the intended behaviour and to the ever-changing demands of the context in which activities unfold. For example, a simple arm extension is sufficient to successfully pick up a cup that is within reach, but trunk and arm movements have to be coordinated if the cup is further away. Relatedly, very different patterns of movement would have to be assembled to pick up a cup of hot coffee from the hands of a well-trained waiter than to recover it from the hands of a young child. Thus, to achieve desired functional goals, humans (and animals) must organize and reorganize action patterns in response to contextual constraints defining the available opportunities for action.

Action selection is shaped by both hard and soft constraints (Fajen, 2007). Hard constraints are factors that define limits on how an action can be performed. For instance, if a driver wishes to stop her car before hitting the garage wall, she must initiate braking early enough to stop with (at least) a minimal space between the car and the wall. The latest time at which braking must start illustrates a hard constraint, defined by the dynamic relationship between velocity, distance to the wall, and maximal deceleration rate. A hard constraint defines an affordance boundary, here the boundary of brake-ability. If this boundary is crossed, task failure results. Within the boundary imposed by this constraint, however, there are multiple ways to successfully accomplish the task: One might start pressing the brake early and decelerate smoothly, or wait until the last minute and press the brake maximally coming to an abrupt stop, or any combination of the two. Factors that shape these decisions are considered soft constraints and may include mood, goals, habits, ability, distractions, need for safety, and so on.

Organism-environment relations that define affordance boundaries, and hence, constitute hard constraints on selection of action patterns have been identified for a variety of tasks, such as climbing, reaching, and grasping (Carello et al., 1989; Cesari \& Newell, 1999; Richardson et al., 2007; Warren, 1984). Individuals show sensitivity to affordance boundaries and organize their action patterns accordingly (Carello et al., 1989). The role of soft constraints on 
Studies in Perception \& Action XV

L. van Dijk \& R. Withagen (Eds.)

action selection is much less studied despite its potential for explaining different levels of performance in underconstrained tasks, that is, tasks allowing for more than one solution for successful completion. Nordbeck et al. (2019) have recently proposed a novel experimental paradigm to focus on this issue. In this experiment, participants transported balls between a starting location and a large wooden box located $9 \mathrm{~m}$ away. Participants' action patterns were assessed under variations in the temporal interval of ball presentation. A variety of action patterns were expected and observed (moving all the way up to the box and dropping the ball, throwing the ball from afar, and some combination of the two). A single task dynamic with two parameters (formalized by the Cusp Catastrophe model; Thom, 1975), however, was able to capture the wide range of participant responses to contextual change (i.e. presentation interval) by accounting for the effect of soft constraints (e.g. motivation). The aim of the present study was to assess whether this model is generalizable to a different underconstrained task designed in virtual reality (VR). The overarching goal is to determine whether the Cusp Catastrophe model can provide an appropriate formal basis for the study of soft constraints affecting context sensitivity of action solutions in underconstrained tasks.

\section{Method}

Thirty undergraduate students participated for partial course credit. A virtual environment was created (see Figure 1) where pucks (marked ' 1 ' in Figure 1) were released from a dispenser (marked ' 2 ') and slid out onto the starting area (' 3 '). The bridge ('4') connected the starting area to a goal container (' 5 '). They were informed that their task was to get the pucks into the container on the other side of the bridge and that they could do so in any way they liked (e.g. push the puck all the way to the container, hit the puck from afar, or a combination of pushing and releasing). Participants were also told to avoid letting the pucks stack up in the starting area. Then they were equipped with a HTC Vive headset and controller and lined up with their right leg in front of the bridge. After a verbal countdown the task was started by presenting pucks to participants. After the first block was completed, participants were given the option to have a short break, the presentation rate was reversed and participants again performed the transportation task. Controller position data was continuously recorded throughout the experiment.

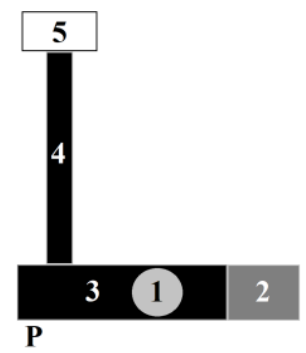

Figure 1. General experimental setup. 
Studies in Perception \& Action XV

L. van Dijk \& R. Withagen (Eds.)

\section{Results and Discussion}

The positional data of the controller was used to create two main variables. Before calculation of the variables, the distance at which participants released each puck was extracted and averaged across each presentation rate for each block. The resulting time-series of release points were then the basis for calculation of the two variables. The first variable took the average of each timeseries, indexing the average distance participants used $(M d)$. The second variable was calculated by finding the largest difference between two consecutive distance points $(\Delta M d)$. Then, the average distance for all distances before this point were subtracted from the ones after and the second variable equalled its absolute value. The two variables were then used to classify the time-series into one of four general solution types that vary in sensitivity to contextual conditions (see Figure 2). Exemplar time-series of the different solutions are plotted in Figure 3 along with comparison solutions (participant and simulated) from Nordbeck et al. (2019).

The reaching task in VR produced highly variable action patterns that could be classified into four general classes of solutions, similar to the ones found in Nordbeck et al. (2019) with the exception that the most extreme ends were not found in this particular setup. Also, the time-series mimicked those of the previous task as well as the model simulations. Results support the utility of the Cusp Catastrophe model in research designed to study the role of soft constraints on affordance actualization. Importantly, the presented research opens up the possibility to explore the versatility of VR in the identification of factors modifying individuals' sensitivity to soft constraints, and how this sensitivity might explain different levels of performance in underconstrained tasks.

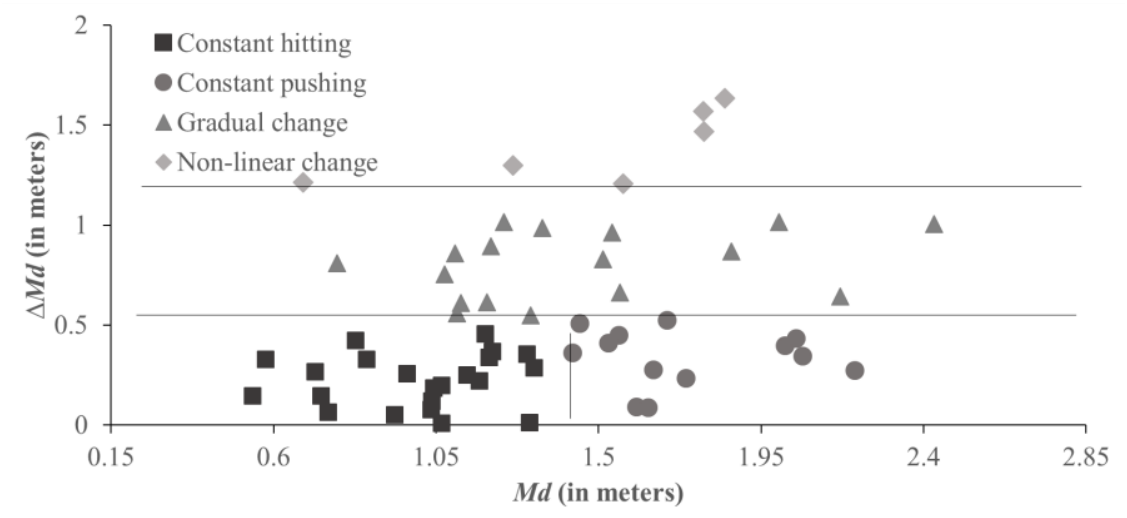

Figure 2. Participant data behaviourally classified as a function of mean distance moved $(M d)$ and difference in mean pre-post max change in distance $(\Delta M d)$. Note. In Nordbeck at al. (2019), behaviours characterized by low $M d \&$ low $\triangle M d$ map on to constant throwing and those characterized by high $M d \&$ low $\triangle M d$ map on to constant walking. 
Studies in Perception \& Action XV

L. van Dijk \& R. Withagen (Eds.)
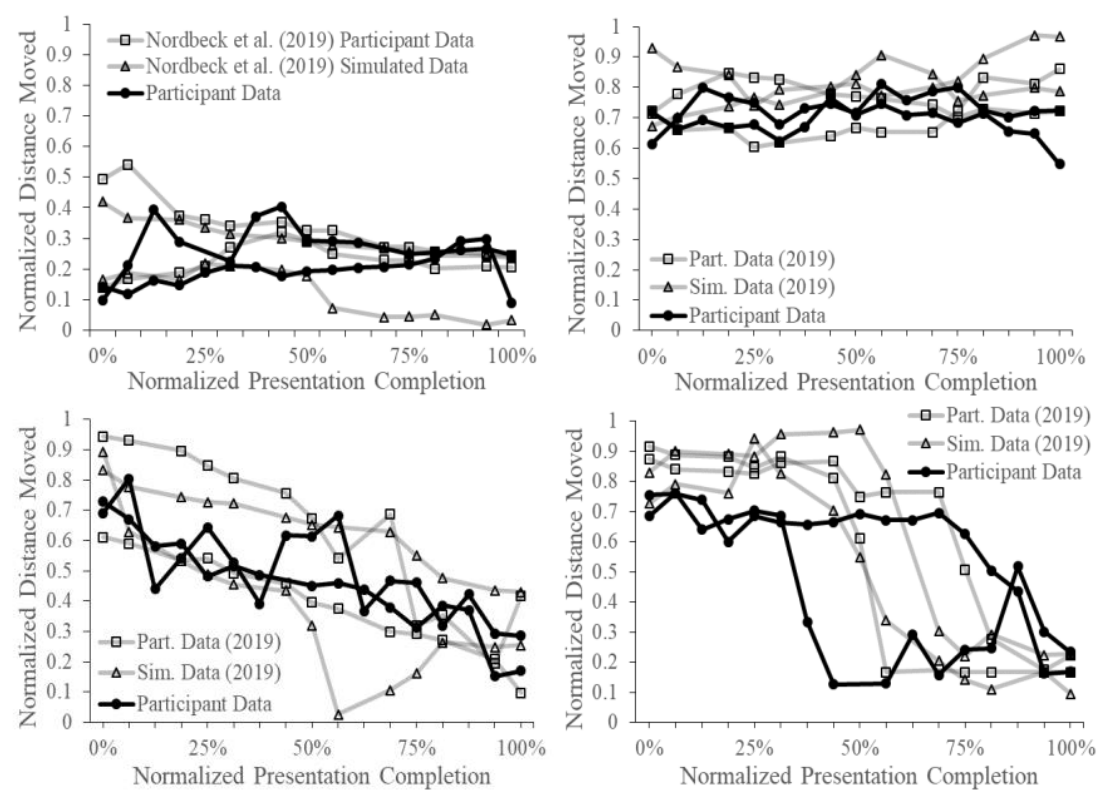

Figure 3. Each figure illustrates a particular behavioral pattern: Low Md \& Low $\Delta M d$ (top left), High $M d \&$ Low $\Delta M d$ (top right), gradual (bottom left), and nonlinear (bottom right), and contains two examples of each data type: behavioral (transparent line, square markers) and simulated data (transparent line, triangle markers) from Nordbeck et al. (2019), and behavioural data from the present research for comparison (full line, circle markers).

\section{References}

Carello, C., Grosofsky, A., Reichel, F. D., \& Solomon, H. Y. (1989). Visually perceiving what is reachable. Ecological Psychology, 1, 27-54.

Cesari, P., \& Newell, K. M. (1999). The scaling of human grip configurations. JEP:HPP, 25, 927-935.

Bernstein, N. (1967). The coordination and regulation of movements. Oxford: Pergamon Press.

Fajen, B. R. (2007). Affordance-based control of visually guided action. Ecological Psychology, 19, 383-410.

Nordbeck, P. C., Soter, L. K., Viklund, J. S., Beckmann, E. A., Kallen, R. W., Chemero, A. P., \& Richardson, M. J. (2019). Effects of task constraint on action dynamics. Cognitive Systems Research, 55, 192-204.

Richardson, M. J., Marsh, K. L., \& Baron, R. M. (2007). Judging \& actualizing intrapersonal and interpersonal affordances. JEP:HPP, 33, 845-859.

Thom, R. (1975). Structural stability and morphogenesis. Reading, MA: W.A. Benjamin.

Warren, W. H. (1984). Perceiving affordances: Visual guidance of stair climbing. JEP:HPP, 10, 683-703. 